\title{
PVDF/Ni fibers synthesis by solution blow spinning technique
}

\author{
Y. J. Dias ${ }^{1}$ - T. C. Gimenes ${ }^{1}$ - S. A. P. V. Torres ${ }^{1}$ - J. A. Malmonge ${ }^{1} \cdot$ A. J. Gualdi ${ }^{2}$ \\ F. R. de Paula ${ }^{1}$
}

Received: 20 July 2017 / Accepted: 30 September 2017 / Published online: 9 October 2017

(c) Springer Science+Business Media, LLC 2017

\begin{abstract}
The development of polymeric fibers incorporated with nickel (Ni) nanoparticles (NPs) has gained broad interest due to its applications, mainly for impurities removal from oil with the aid of magnetic field. In the present study, poly(vinylidene fluoride) (PVDF) fibers containing Ni NPs were produced by solution blow spinning (SBS) technique. Scanning electron microscope (SEM) measurements showed fibers with an average diameter of $564 \mathrm{~nm}$, randomly dispersed without a preferred orientation. X-ray spectrum shows peaks related to Ni showing that Ni NPs were incorporated into the fibers. The magnetic analysis of the nanocomposite shows the hysteresis loops typical of ferromagnetic behavior and ZFC-FC curves revealed the presence of superparamagnetic behaviour and an average blocking temperature of $165 \mathrm{~K}$. The results achieved in this work showed that is possible to produce fibrous material, using SBS technique, with unusual magnetic properties that may be applicable in various fields, including different areas of technological industry.
\end{abstract}

\section{Introduction}

Nanoscale materials have attracted broad interest in the last decade due to the impact them may cause in science. This technologic branch is named nanotechnology, aims to

F. R. de Paula

depaula@dfq.feis.unesp.br

1 Faculdade de Engenharia, Universidade Estadual Paulista (UNESP), Câmpus de Ilha Solteira, Ilha Solteira, SP 15385-000, Brazil

2 Grupo de Supercondutividade e Magnetismo, Departamento de Física, Universidade Federal de São Carlos, São Carlos, SP CEP 13565-905, Brazil control the fundamental structure and behavior of matter, reaching atomic and molecular levels. This domain offers the possibility to interpret new phenomena and create new properties presented in micro and nanoscopic scales. These characteristics might offer excellent and differentiated functions comparing to bulk materials. Nanoscale materials properties are strongly influenced by the surface (ratio area/volume), being equivalent or superior to volume effects. Changes in chemistry reaction, electric conductivity, enhancement of resistance, and loss of magnetic properties are observed when materials dimensions are $<100 \mathrm{~nm}$. Potential areas for nanomaterials include areas like nanoelectronics, nanofiltration, and nanosensors [1-5].

In the development of nanomaterials, polymeric fibers are in expansion in different knowledge areas. There are many techniques to synthesize polymeric fibers like wet spinning, dry spinning, melt spinning, gel spinning and, among others. Nowadays the most popular is electrospinning, which utilizes electrical forces to produce polymer fibers with diameters ranging from a few nanometers to several micrometers. However, a technique recently developed named solution blow spinning (SBS) was also succeeded [6-10]. Compared to electrospinning process, SBS does not use high voltage field, the production rate is several times higher with fiber diameters similar to those produced by electrospinning. The SBS apparatus is formed by a concentric nozzle, compressed air (nitrogen, argon or air), pressure measurer, a hypodermic syringe, a system of mechanical injection, and a rotator collector with controlled velocity, as shown in Fig. 1.

In the SBS process, the solution is pumped through the inner nozzle with a fixed injection ratio. The concentric geometry of the nozzle favors the solution to adopt a conical shape at the end of the needle due to air flux which is responsible for dragging the fibers to the collector. Along with its way, the solvent can be wholly or partially evaporated, and 


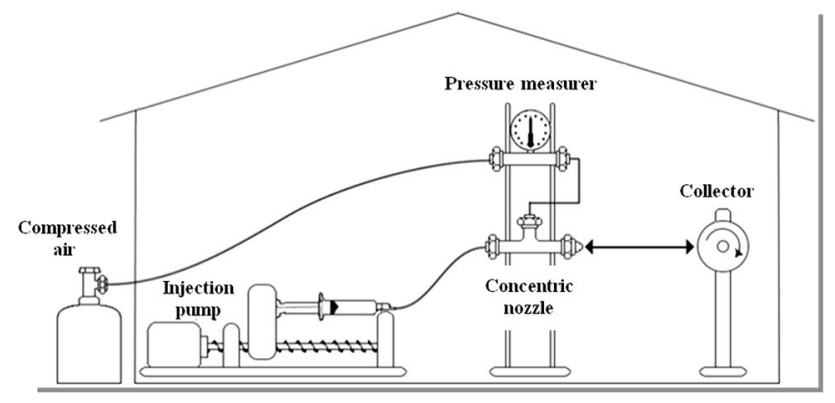

Fig. 1 Experimental apparatus used by SBS technique [1]

the fibers are stretched and usually collected by the rotating collector.

To produce fibers containing Ni NPs, PVDF was chosen as a base material because of its excellent properties as high mechanical strength and good resistance to solvents, acids, and bases, high dielectric constant and piezoelectric properties. Another important characteristic is that PVDF is a hydrophobic polymer and when incorporated with magnetic nanoparticles which have a high affinity for oil, may allow its easy removal [11-13]. Nickel is an example of a magnetic particle that has been investigated as a metal remover. As it is well known, Ni is a ferromagnetic FCC metal with a Curie temperature of $627 \mathrm{~K}$ with easy magnetization axis $<111>$ and $<100>$ hard.

In this paper, a simple methodology to fabricate PVDF fibers contained Ni NPs was introduced. The nanocomposite was characterized by Scanning Electron Microscopy (SEM), X-ray Diffraction (XRD) and MPMS3 SQUID magnetometer. The results showed that the PVDF/Ni fibers obtained exhibit ferromagnetic behavior and can be applied in various technology sectors where the magnetic properties are required.

\section{Materials and methods}

\subsection{Chemicals and materials}

The polymer used as the matrix was PVDF (Foraflon 4000 HD) and the Ni NPs were purchased from Atochem and Sigma Aldrich, respectively. Dimethylformamide (DMF) was purchased from Synth and was used without further treatment.

\subsection{Sample preparation}

The solution was prepared by mixing $5 \mathrm{~mL}$ of DMF with $1 \mathrm{~g}$ of PVDF. The mixture was vigorously stirred at $343 \mathrm{~K}$ for $1 \mathrm{~h}$ by a magnetic stirrer to ensure the dissolution of PVDF. Then, the solution was cooled to room temperature, and $0.1 \mathrm{~g}$ of Ni NPs was added. The mixture was stirred continuously for approximately $15 \mathrm{~min}$ for better $\mathrm{Ni}$ dispersion. To obtain the fibers, $3 \mathrm{~mL}$ of the PVDF/Ni dispersion was placed in a disposable syringe (connected to inner nozzle) coupled to the injection system. The best conditions found to perform the SBS were: work distance (nozzle/collector) of $22 \mathrm{~cm}$; the gas (air) pressure of $76 \mu \mathrm{L} / \mathrm{min} 140 \mathrm{kPa}$; collector speed of $400 \mathrm{rpm}$ and dispersion injection rate of $76 \mu \mathrm{L} / \mathrm{min}$.

\subsection{Characterizations}

The structures of the samples were studied by X-ray Diffraction by SHIMADZU XRD-6000 model which provides $\mathrm{Cu} \mathrm{K} \alpha$ radiation $(\lambda=1.54 \AA)$. Measurements were taken in $\theta-2 \theta$ configuration from $5^{\circ}$ at $80^{\circ}$, with a step size of $0.02^{\circ}$. Scanning electron microscope (SEM) images were obtained with ZEISS model EVO LS15 with acceleration voltage of $0.1-20 \mathrm{kV}$ coupled with EDX analyzer for stoichiometry. The samples were attached to aluminum stubs with conductive carbon tape and sputtered with gold before analysis. The fibers diameters were determined by ImageJ $1.45 \mathrm{~s}$, software. Magnetic characterizations were performed by using Magnetometer-SQUID-VSM (MPMS 3 - Quantum Design). Magnetization measurements were performed as a function of applied magnetic field at 5 and $300 \mathrm{~K}(M \times H)$. Because of the hysteretic effect of the sample studied, both field cooled (FC) and zero-field cooled (ZFC) measurements have been performed. FC measurements are done when the sample is cooled in the field at room temperature, and in ZFC measurements, the sample is first cooled to a low temperature in zero fields and a field is then applied.

\section{Results and discussion}

To ensure that the added Ni NPs are really present in the polymeric fibers, energy-dispersive X-ray spectroscopy (EDX) was utilized. The results are presented in Fig. 2. The carbon $(0.27 \mathrm{keV})$, and fluorine $(0.67 \mathrm{keV})$ peaks correspond to the presence of PVDF. The signals at 0.87, 7.49 and $8.29 \mathrm{keV}$ represent $\mathrm{Ni}$, demonstrating the successful incorporation of metal in the fibers.

Figure 3 shows X-ray diffraction analysis of the sample containing $0.1 \mathrm{~g}$ of Ni NPs and SEM images of the PVDF/ Ni fibers. The XRD spectra (Fig. 3a) show peaks located at $44.56^{\circ}$ and $51.92^{\circ}, 76.55^{\circ}$ and 93.01 corresponding to the planes (111) and (200), (220) and (311), respectively (ICSD 64989 PDF $87-712$ ). The peaks located at $18.40^{\circ}$ and $20.60^{\circ}$, corresponding to the planes (020) and (100), are related to $\alpha$ and $\beta$ phase, respectively, of PVDF [14]. The SEM image (Fig. 3b) shows fibers with a circular cross-section with an average diameter of $564 \mathrm{~nm}$ with smooth surfaces and free 


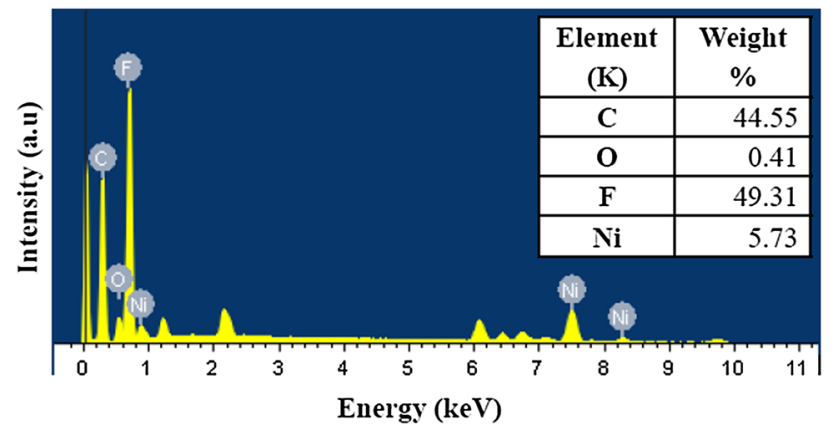

Fig. 2 EDX spectrum of the PVDF/Ni fibers

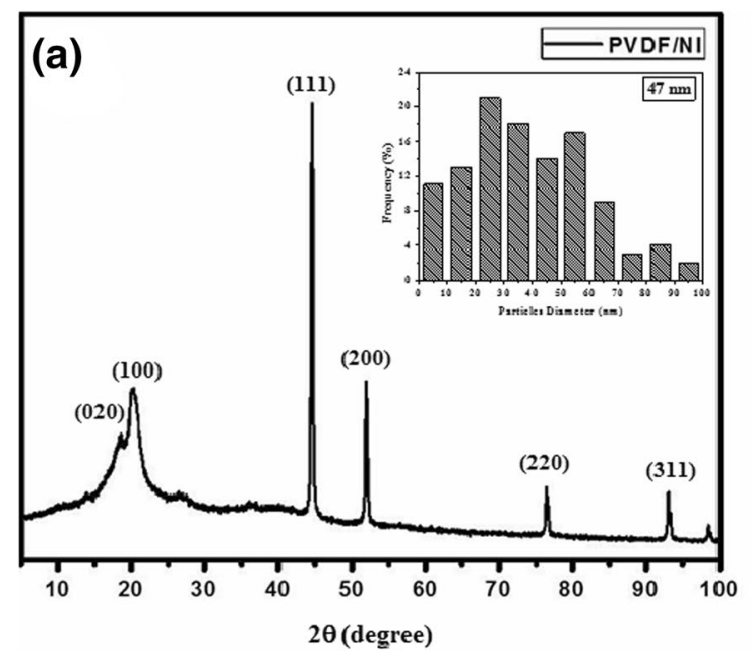

of beads. The Ni NPs in the fibers were not observed, this result is an indication that they are inside of fibers.

The magnetic properties of the PVDF/Ni fibers have been studied. Figure $4 \mathrm{a}$ shows the hysteresis loops at 5 and $300 \mathrm{~K}$, Fig. 4b shows details of magnetization at low field. Since the PVDF is not magnetic material, the magnetic properties of the produced fibers strongly rely on the contents of magnetite nanoparticles. One can observe that the fibers reveal typical ferromagnetic behavior. This behavior is an indication of the presence of exchange coupling among particles due to particle aggregation.

The ferromagnetism in the fibers is shown by coercivity $\left(H_{c}\right)$, saturation magnetization $\left(M_{s}\right)$, a remanent magnetization $\left(M_{r}\right)$, and saturation field $\left(H_{s}\right)$. These quantities are listed in Table 1 . At $5 \mathrm{~K}$ the fibers exhibit greater $H_{c}$ than at $300 \mathrm{~K}$, at room temperature is $43 \mathrm{Oe}$, while at $5 \mathrm{~K}$ is

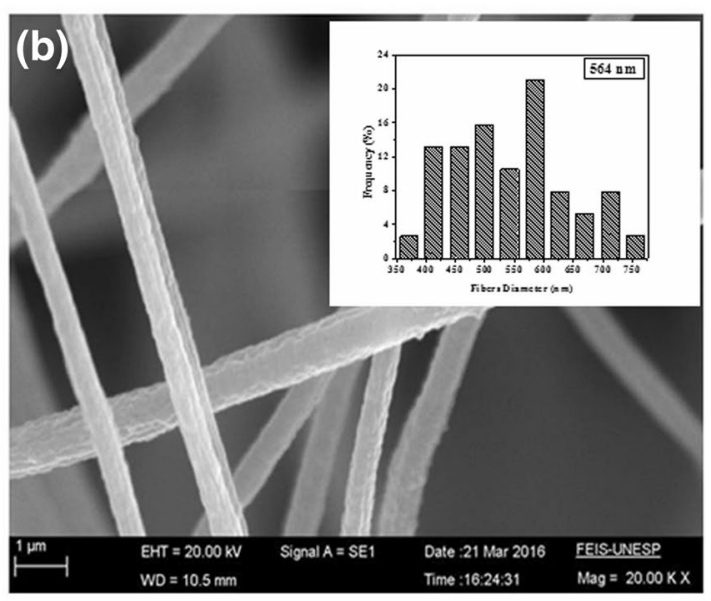

Fig. 3 a X-ray diffraction analysis of a sample containing $0.1 \mathrm{~g}$ of Ni NPs (figure inset: Ni particles distribution) and $\mathbf{b}$ SEM images of the PVDF/Ni fibers
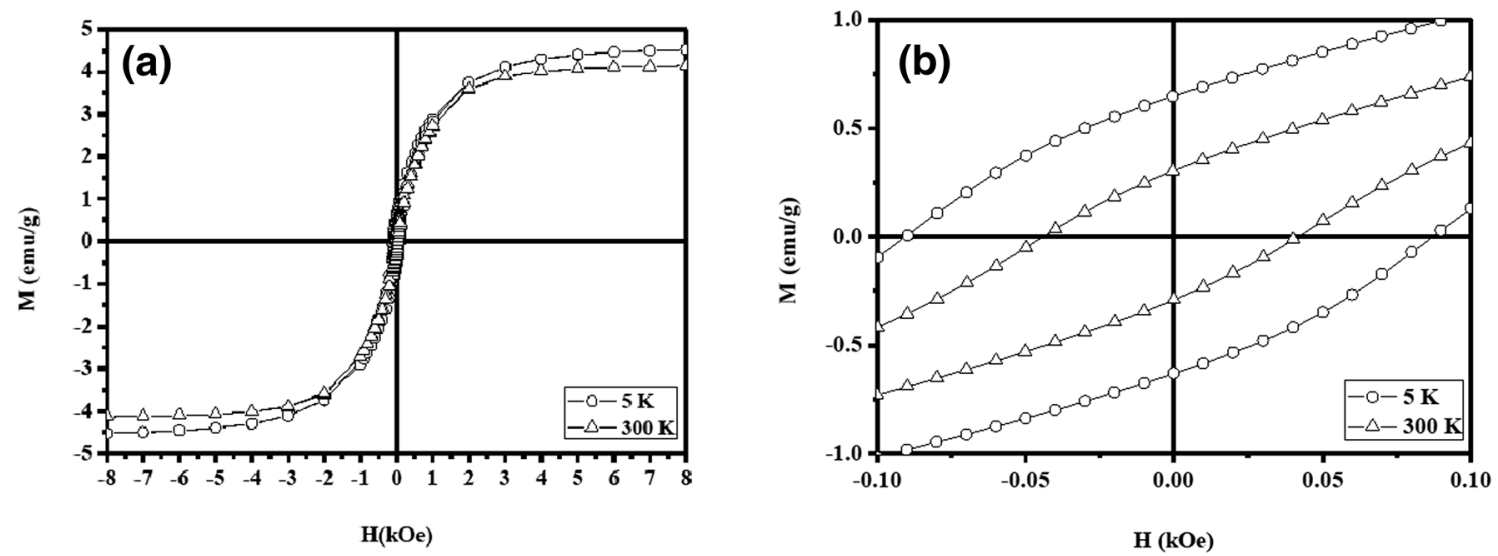

Fig. 4 a Magnetic curve in function of applied magnetic field $(M \times H)$ running in the sample incorporated with $0.1 \mathrm{~g}$ of Ni NPs under a temperature of 5 and $300 \mathrm{~K}$. b Shows details of magnetization at low field 
Table 1 Magnetic parameters at a temperature of 5 and $300 \mathrm{~K}$

\begin{tabular}{lll}
\hline Parameter & $5 \mathrm{~K}$ & $300 \mathrm{~K}$ \\
\hline Coercivity, $\mathrm{H}_{\mathrm{c}}(\mathrm{kOe})$ & 90 & 43 \\
Saturation magnetization, $\mathrm{M}_{\mathrm{s}}(\mathrm{emu} / \mathrm{g})$ & 4.5 & 4.1 \\
Remanent magnetization, $\mathrm{M}_{\mathrm{r}}(\mathrm{emu} / \mathrm{g})$ & 0.64 & 0.30 \\
Saturation field, $\mathrm{H}_{\mathrm{s}}(\mathrm{kOe})$ & 8 & 8 \\
\hline
\end{tabular}

90 Oe. The largest coercivity at a lower temperature may be explained by considering the reduced influence of thermal fluctuation on the rotation of magnetic dipoles. At $5 \mathrm{~K}$ is required an increased amount of energy (or higher magnetic field strength) to change the magnetization direction of theses aligned dipoles, and therefore a large coercivity of the sample can be observed. It is also seen in Table 1 that $M_{r}$ and $M_{s}$ obtained at $5 \mathrm{~K}$ are greater than compared at $300 \mathrm{~K}$. In this case at high temperature the contribution of the particles in the superparamagnetic state can be larger than at $5 \mathrm{~K}$.

Figure 5 shows the temperature dependence of magnetization, $M(T)$ on the application of 100 (Fig. 5a) and $1000 \mathrm{Oe}$ (Fig. 5b) in ZFC and FC cycles in the temperature range 5-300 K. The ZFC-FC curves show an irreversible magnetic behavior and dependent on the intensity of the applied magnetic field. The ZFC-FC curves of the sample with an external magnetic field of 100 Oe, measured in the temperature range of 5-300 $\mathrm{K}$ do not overlap at $300 \mathrm{~K}$. This behavior indicates the occurrence of superparamagnetic particles together with a fraction of nanoparticles still in a blocked magnetic state at room temperature. This behavior can be due to the large mean size of nanoparticles, but also to the presence of strong interparticle interactions [15]. The ZFC-FC curves obtained at 1000 Oe shows the typical superparamagnetic behavior. Around $250 \mathrm{~K}$ the $\mathrm{ZFC}-\mathrm{FC}$ curves start to separate, this behavior is evidence of the blocking of the largest particles. At the temperature of $165 \mathrm{~K}$, the curve maximum of $\mathrm{ZFC}$ is observed representing average blocking temperature.

\section{Conclusions}

In conclusion, we have developed a new strategy for the facile production of fibers with magnetic behavior. Fibers of PVDF/Ni with smooth surfaces, free of beads and with an average diameter of $564 \mathrm{~nm}$ were prepared by Solution Blow Spinning technique. The $M \times H$ measurements showed ferromagnetic behavior, under the temperature of 5 and $300 \mathrm{~K}$ respectively, coercive field of 90 and $43 \mathrm{Oe}$, and remanence of 0.64 and $0.30 \mathrm{emu} / \mathrm{g}$. The $\mathrm{ZFC}-\mathrm{FC}$ curves revealed the presence of superparamagnetic behavior and an average blocking temperature of $165 \mathrm{~K}$. Also, the preparation of PVDF fibers with an incorporation of Ni by SBS technique can be extended to various types of magnetics nanoparticles. These magnetic fibers can potentially be used in the fabrication of magnetic sensors, flexible magnets, spintronic devices and process of removing impurities from oil, water, and blood.

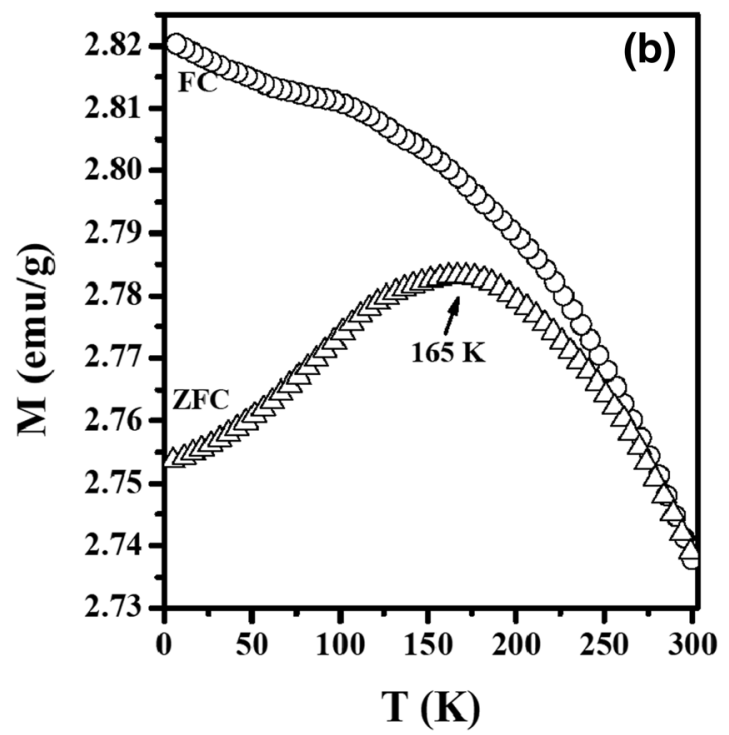

Fig. 5 ZFC-FC magnetization recorded with an external magnetic field of a 100 Oe and b 1000 Oe 
Acknowledgements We thank for FAPESP, CNPq and CAPES for their financial support. We also thank the SQUID-UFSCar (FAPESP 09/54082-2) for the magnetic measurements.

\section{References}

1. T. Thurn-Albrecht, J. Schotter, G.A. Kastle, N. EmLey, T. Shibauchi, L. Krusin-Elbaum, K. Guarini, C.T. Black, M.T. Tuominen, T.P. Russell, Ultrahigh-density nanowire arrays grown in selfassembled diblock copolymer templates. Science 290, 2126-2129 (2000)

2. D.A. Allwood, G. Xiong, M.D. Cooke, C.C. Faulkner, D. Atkinson, N. Vernier, R.P. Cowburn, Submicrometer ferromagnetic not gate and shift register. Science 296, 2003-2006 (2002)

3. J.I. Martín, J. Nogués, K. Liu, J.L. Vicent, I.K. Schuller, Ordered magnetic nanostructures: fabrication and properties. J. Magn. Magn. Mater. 256, 449-501 (2003)

4. A. Fert, L. Piraux, Magnetic nanowires. J. Magn. Magn. Mater. 200, 338-358 (1999)

5. L.M. Lu, L. Zhang, F.L. Qu et al., A nano-Ni based ultrasensitive nonenzymatic electrochemical sensor for glucose: enhancing sensitivity through a nanowire array strategy. Biosens. Bioelectron. 25, 218-223 (2009)

6. R. Maria, R. Rodrigues, J. Elvis, E. Medeiros, Production of submicrometric fibers of mullite by solution blow spinning (SBS). Mater. Lett. 149, 47-49 (2015)

7. B. Cheng, X. Tao, L. Shi, G. Yan, X. Zhuang, Fabrication of $\mathrm{ZrO}_{2}$ ceramic fiber mats by solution blowing process. Ceram. Int. 40, 15013-15018 (2014)
8. E.S. Medeiros, G.M. Glenn, A.P. Klamczynski, W.J. Orts, L.H.C. Mattoso, Solution blow spinning: a new method to produce microand nanofibers from polymer solutions. J. Appl. Polym. Sci. 113, 2322-2330 (2009)

9. S. Afonso, J.E. Oliveira, E.A. Moraes, RGF Costa, LHC Mattoso, W.J. Orts, E.S. Medeiros, W. Luis, Nano and submicrometric fibers of poly(D,L-lactide) obtained by solution blow spinning: Process and solution variables. J. Appl. Polym. Sci. 122, 3396-3405 (2011)

10. M. Rotta, L. Zadorosny, C.L. Carvalho, J.A. Malmonge, L.F. Malmonge, R. Zadorosny, YBCO ceramic nanofibers obtained by the new technique of solution blow spinning. Ceram. Int. 42, 16230-16234 (2016)

11. F. Byrne, A. Prina-Mello, A. Whelan et al., High content analysis of the biocompatibility of nickel nanowires. J. Magn. Magn. Mater. 321, 1341-1345 (2009)

12. P.G. Tratnyek, R.L. Johnson, Nanotechnologies for environmental cleanup. Nanotoday 1, 44-48 (2006)

13. N. Savage, M.S. Diallo, Nanomaterials and water purification: opportunities and challenges. J. Nanopart. Res. 7, 331-342 (2005)

14. S. Yu, W. Zheng, W. Yu, Y. Zhang, Q. Jiang, Z. Zhao, Formation mechanism of $\beta$-phase in PVDF/CNT composite prepared by the sonication method. Macromolecules 42, 8870-8874 (2009)

15. D. Fiorani, J.L. Dormann, R. Cherkaoui, E. Tronc, F. Lucari, F. D’Orazio, L. Spinu, M. Nogus, A. Garcia, A.M. Testa, Collective magnetic state in nanoparticles systems. J. Magn. Magn. Mater. 196-197, 143-147 (1999) 